\title{
EchoGéo
}

26 | 2013

Varia

\section{Travail et espace dans la global food. Expériences des saisonnières agricoles entre domination, reconnaissance et émancipation}

Entretien avec Djemila Zeneidi à propos de la parution de son ouvrage Femmes/fraises, import/export, Paris, PUF, coll. Souffrance et théorie, 2013

\section{Albena Tcholakova}

\section{(2) OpenEdition}

\section{Journals}

Édition électronique

URL : https://journals.openedition.org/echogeo/13671

DOI : 10.4000/echogeo.13671

ISSN : 1963-1197

Éditeur

Pôle de recherche pour l'organisation et la diffusion de l'information géographique (CNRS UMR 8586)

Référence électronique

Albena Tcholakova, «Travail et espace dans la global food. Expériences des saisonnières agricoles entre domination, reconnaissance et émancipation », EchoGéo [En ligne], 26 | 2013, mis en ligne le 19 décembre 2013, consulté le 31 juillet 2021. URL : http://journals.openedition.org/echogeo/13671 ; DOI : https://doi.org/10.4000/echogeo.13671

Ce document a été généré automatiquement le 31 juillet 2021.

EchoGéo est mis à disposition selon les termes de la licence Creative Commons Attribution - Pas d'Utilisation Commerciale - Pas de Modification 4.0 International (CC BY-NC-ND) 


\section{Travail et espace dans la global food. Expériences des saisonnières agricoles entre domination, reconnaissance et émancipation}

Entretien avec Djemila Zeneidi à propos de la parution de son ouvrage Femmes/fraises, import/export, Paris, PUF, coll. Souffrance et théorie, 2013

\section{Albena Tcholakova}

1 L'entretien a été conduit à Paris, le 28 novembre 2013 par Albena Tcholakova.

2 Djemila Zeneidi, djemila.zeneidi@cnrs.fr, est chargée de recherche au CNRS - ADESS UMR Aménagement, Développement, Environnement, Santé et Sociétés - UMR 5185. Elle a notamment publié :

- Zeneidi D., 2002. Les SDF et la ville. Géographie du savoir-survivre. Paris, Bréal, coll. D'autre part.

- Zeneidi D., 2013. Femmes/fraises, import/export. Paris, PUF, coll. Souffrance et théorie.

3 - Albena Tcholakova (AT). Djemila Zeneidi, votre dernier livre avec un titre original ne nous laisse pas indifférent. Il rend compte d'une enquête de moyenne durée, menée auprès de femmes marocaines ouvrières agricoles de ce que l'on appelle de plus en plus la global food. "Ici », il s'agit des plantations de fraises, dans la province espagnole d'Huelva et plus précisément à Cartaya où nous suivons ces nouvelles ouvrières migrantes. "Là-bas ", c'est à Rabat et Kénitra, au Maroc que vous avez mené votre recherche $^{1}$. Celle-ci interroge la fabrication d'un système migratoire du travail temporaire des "contrats en origine", donné en exemple de "gouvernance» migratoire, et de « rationalisation » du travail, par plusieurs institutions européennes. Le livre est un travail sur les espaces globalisés et précaires du travail global et précaire qui met en exergue, avec une approche théorique originale, plusieurs enjeux contemporains liés aux migrations et au travail. 
4 Djemila Zeneidi, vous nous amenez au cœur de l'émergence de nouveaux espaces productifs où domination et émancipation se conjuguent de manière ambivalente, et où le travail occupe une place centrale dans l'expérience spatiale des femmes ouvrières migrantes et dans la construction du rapport positif à elles-mêmes. Pourriez-vous revenir sur le contexte de votre enquête, en nous décrivant le dispositif d'encadrement de la « contractualisation en origine »?

5 - Djemila Zeneidi (DZ). La contractualisation en origine, que l'on appelle aussi contractualisation à la source est un dispositif de recrutement des migrants temporaires. Cela implique que le recrutement et la signature du contrat se font dans le pays de départ. Le dispositif de recrutement est mis en place par la loi espanole (La ley de extranjerià) qui régit l'entrée et le séjour des étrangers (4/2000).

6 Par ailleurs, ce dispositif d'encadrement et de gestion de cette main-d'œuvre s'applique aux ouvrières marocaines recrutées via les contrats en origine, à l'initiative de Cartaya ville de la province de Huelva, qui a saisi l'opportunité de financement de l'Union européenne. Le dispositif est en adéquation avec les préconisations de l'Union européenne, qui fait une promotion active de la migration temporaire ou circulaire et de son management. La lutte contre l'immigration illégale est une préoccupation majeure. Il s'agit d'empêcher l'installation définitive des migrants sur le sol européen. Le système d'encadrement prend en charge les ouvrières depuis le Maroc jusqu'à leur retour dans le pays. Il couvre le transport, le séjour et le retour. Un certain nombre d'employés de la mairie dont une partie de médiateurs marocains (employés vacataires) sont chargés du suivi des ouvrières ; ils sont affectés à des fermes.

7 - AT. Comment s'est déroulée l'enquête elle-même? Quel-l-e-s sont les différent-e-s acteur-rice-s que vous avez rencontré-e-s?

8 - DZ. Au Maroc, j'ai rencontré des acteurs institutionnels de l'agence marocaine de l'emploi (Anapec), de l'Ambassade d'Espagne et des ouvrières dans leur famille dans la ville de Kénitra et dans le douar de Kreda (province de Kenitra).

9 En Espagne, j'ai rencontré différents responsables et employés de la mairie de Cartaya aux services de l'immigration, du logement. J'ai rencontré les membres d'un syndicat proche du patronat, qui est partie prenante de l'encadrement. J'ai par ailleurs rencontré des employés et le responsable d'une structure associative spécialisée dans l'accueil des migrants. Cette association est prestataire de service, joue un rôle dans le suivi des ouvrières car elle dispense des cours d'hygiène, d'éducation sexuelle, «d'incitation au retour» et gère les cas de femmes enceintes ou qui viennent d'accoucher. Les employeurs: j'en ai rencontré quelques-uns, mais ce fut très compliqué. C'était dans le cadre de visite de fermes modèles, mises en scène pour les visiteurs extérieurs, visites organisées par la mairie. Ils assimilaient les chercheurs aux journalistes.

10 Cette attitude de méfiance s'est généralisée et étendue aux acteurs institutionnels qui étaient au début de mon enquête fin 2009 début 2010 plutôt ouverts et fiers de présenter le fonctionnement du dispositif. Elle est apparue plus tard, liée à des critiques relayées par les médias. Les employés de la mairie m’ont expliqué que les réticences que je rencontrais étaient à la suite de la diffusion sur une chaîne française d'un documentaire (La rançon de la fraise). Ils ont laissé la réalisatrice faire son travail et la sortie du documentaire a rendu les employeurs furieux. Dans ce contexte, les employeurs ne se bousculaient pas pour ouvrir les portes de leurs exploitations. 
11 J'ai voulu rencontrer les ouvrières par moi-même dans les fermes et dans les foyers d'hébergement de saisonnier(e)s. Là, je me suis rendue compte du contrôle exercé sur ces ouvrières soumises à une surveillance très stricte, voire une forme de captivité. Elles ne pouvaient pas par exemple recevoir de visites. Il a fallu adapter la méthode d'enquête et aller à la rencontre de ces ouvrières sur leurs trajets pour faire leurs courses, dans la rue, dans les commerces. Ce qui comporte beaucoup de limites, mais je n'avais pas le choix.

12 - AT. Votre analyse mobilise une approche multiscalaire de l'espace et plus largement de l'expérience des ouvrières migrantes. Pourquoi ce choix?

13 - DZ. Cette expérience est en effet produite à l'intersection de plusieurs échelles. La première échelle est celle d'une agriculture globalisée, issue du modèle californien, agriculture destinée à l'exportation. En termes de contrôle migratoire, l'Union européenne, le Maroc, l'État espagnol, la Communauté Autonome d'Andalousie, la province de Huelva, les villes et réseaux de villes concernés par l'immigration, participent à différents degrés à la construction de la contractualisation en origine et du dispositif de gestion. Il y a là des enjeux de gouvernance importants, surtout lorsque l'on voit que la politique migratoire prend appui sur l'échelon local.

14 Ce contexte socio-économique et politique multiscalaire contribue forcément à déterminer l'expérience des ouvrières et cela produit des formes de subjectivation et également de résistance spécifiques au pouvoir...

15 Cette expérience est aussi une expérience corporelle. Ce qui m'a intéressé, c'est de voir comment jusque dans leur corps les ouvrières sont affectées par cette politique et cette économie produite à des niveaux différents.

16 - AT. Les femmes migrantes marocaines, ouvrières agricoles, que vous avez rencontrées occupent une place souvent spécifique dans le système migratoire européen, qui ne saurait se réduire ni au seul dispositif spatial local, ni à la seule expérience de la domination. Pourriez-vous justement nous expliquer ces ambivalences entre espace, domination et reconnaissance?

17 - DZ. Ce qui m'a frappé dès les premiers entretiens, c'est vraiment cette réalité complexe de l'expérience, et quelque chose qui surgit de l'ordre de la modification, permise par le travail et la migration qui peut émanciper malgré des aspects très négatifs du travail comme la pénibilité par exemple. Se dégageait des propos tenus par ces ouvrières un très fort désir de travailler en Espagne, d'être mobile, et cela tout en admettant qu'il y avait des choses difficiles à vivre dans ce travail. J'ai essayé de comprendre ce qui articulait la dimension négative de cette situation d'ouvrière de seconde zone et les aspects de ce travail et plus largement de la mobilité, apparaissant dans leurs propos comme émancipateurs. Cela s'exprimait en termes de mépris, de rapports positifs à soi, donc dans des catégories de reconnaissance. Par ailleurs, ces ouvrières avaient des attentes précises vis-à-vis de l'employeur. Elles espéraient obtenir un statut de résidente permanente. Pour cela, il fallait obtenir de l'employeur un contrat de travail. Cette promesse de reconnaissance était le ressort de leur consentement. Le management des ouvrières prenait appui sur cette attente de reconnaissance, qui n'était qu'une fausse reconnaissance car elles ne pouvaient obtenir «les papiers » qu'on leur faisait miroiter, ne remplissant pas les conditions requises, mais cela, elles l'ignoraient. Ainsi la reconnaissance se présentait dans ce cas comme un outil de gestion de la main-d'œuvre et aussi de la migration. 
18 Quelle est la nature du lien entre espace et reconnaissance ? Elle est dialectique. L'espace se présente comme une des modalités et supports de la reconnaissance sous toutes ses formes. À l'écoute de ces ouvrières, on se rend compte que ces rapports positifs à soi ou au contraire la dévalorisation étaient concrets, et liés à des pratiques spatiales, à la matérialité même de l'espace. Cela révèle aussi la dimension de médiation, de qualification et relationnelle de l'espace. Ainsi j'ai mobilisé la théorie de la reconnaissance d'Axel Honneth $(2000 ; 2006)$, en introduisant davantage la dimension matérielle que je viens d'évoquer, et les travaux de Jean-Philippe Deranty (2005) et de Emmanuel Renault $(2004 ; 2008)$ ont été dans cette orientation une source d'inspiration importante. En ce qui concerne la dialectique entre expérience de domination et expérience d'émancipation, et cette expérience corporelle dont j'ai parlé plus haut, c'est bien à Foucault $(1975$; 1979) que je me réfère, mais aussi à Natacha BorgeaudGarciandía (2009), à Christophe Dejours (1998 ; 2009), et d'autres auteurs qui travaillent dans cet esprit. L'objet du livre était bien d'apporter un éclairage sur une dialectique domination / émancipation, via l'espace. Il me fallait mobiliser des approches géographiques qui pouvaient en rendre compte.

19 - AT. Ceci m'amène justement à la question suivante. Quelle est votre approche de la géographie radicale, de la geography of labor et de la labor geography?

20 - DZ. La géographie en règle générale a quelque chose à dire sur ces expériences de mobilité, sur l'expérience migratoire et sur les inégalités. La géographie radicale permet précisément de penser la domination à partir d'une conceptualisation forte de l'espace, c'est-à-dire qui ne se limite pas à un cadre mais qui soit réellement investi comme un outil pour décoder le fonctionnement du capitalisme à l'origine du type d'agriculture que j'ai étudié. Les travaux comme ceux de David Harvey (2008) ou encore de Don Mitchell $(2011$; 2012) - là je me réfère à ses recherches sur les travailleurs agricoles mexicains employés dans les exploitations californiennes dans le cadre du programme bracero -, permettent de penser les mécanismes généraux de cette économie pour analyser spatialement des processus économiques. La géographie radicale donne des outils très stimulants. Sur le plan de la gouvernance, les mutations en termes de transfert de compétences, des auteurs comme Neil Brenner et Nik Theodore (2002), ont produit à ce sujet des grilles d'analyses qui sont pertinentes.

21 En m'intéressant à cette thématique, à ce courant, j'ai découvert un auteur qui, tout en s'inscrivant dans la géographie radicale, pose la question de la place des travailleurs dans cette géographie des structures. Selon Andrew Herod (1997), la géographie marxiste élude un peu le travailleur, porte un intérêt quasi exclusif au capital, aux entreprises, aux structures. Le capital n'est pas le seul à modeler le paysage dans ce processus de maximisation de profits. Herod a renforcé le rôle du sujet en portant une attention à leur capacité d'action (agency).

22 - AT. Approches matérielles et spatiales de la domination, de la reconnaissance et de son déni, perspectives genrées prenant au sérieux la sexualité dans l'expérience de travail des femmes vous induisent à proposer la figure de l'ouvrière migrante « cyborg ». Pouvez-vous nous en dire un peu plus?

23 - DZ. Cette figure du Cyborg m'a intéressée pour sa puissance évocatrice. En lisant Donna Haraway (2007), j'ai retrouvé des éléments de l'expérience des ouvrières. La fabrication par un système d'une figure d'ouvrière performante, sélectionnée selon des critères physiques, sexuels et d'origines sociales particuliers, a retenu mon attention. Il faut rappeler que les ouvrières pour être recrutées doivent être mères de famille. On 
voit à l'œuvre une typification genrée, ethnique, voire racialisée dans certains discours. Tout cela participe du modelage dans «le laboratoire » du contrôle migratoire et de l'optimisation économique d'une unité ancrée dans de puissantes fictions : «l'ouvrière marocaine ", ouvrière idéale pour une supposée docilité et qui va être fichée, suivie selon le système de traçabilité biométrique préconisé par l'Union européenne.

Pour être considérée comme une bonne ouvrière, et une bonne migrante, l'ouvrière marocaine doit se conformer aux attentes des employeurs et rentrer chez elle à l'issue de la saison. Certaines ne rentrent pas et quittent l'exploitation pour rester sur le territoire espagnol. J'ai trouvé intéressant de porter mon attention sur la transgression des normes imposées, une transgression qui a des conséquences en termes de ce qu'elles sont au regard des autres. Ces ouvrières sont façonnées à l'intérieur d'un cadre moral qui les définit comme des mères de famille. Ne pas rentrer au pays au terme du contrat, c'est la sortie de cette définition de mère de famille et ouvrière responsable définie dans une double relation de soin à l'enfant et de loyauté à l'employeur. Elles sont immédiatement alors vues comme des mauvaises mères et des putains du côté des marocains. C'est là où la figure du Cyborg est stimulante car elle permet de rendre compte, ainsi que l'énonce Donna Haraway, de la capacité à s'affranchir des cadres de la domination et des codes de genre. Assumer la monstruosité symbolisée par la mauvaise réputation est un pari sur elles-mêmes, un pari pour réaliser un puissant désir, celui d'être autonomes.

\section{BIBLIOGRAPHIE}

Brenner N., Theodore N., 2002. Preface: From the new localism to the spaces of neoliberalism. Antipode, p. 341-347.

Borgeaud-Garciandía N., 2009. Dans les failles de la domination, Paris, PUF.

Dejours C., 2009. Travail vivant. Vol. 1 : Sexualité et travail. Paris, Payot.

Dejours C., 1998. Souffrance en France, la banalisation de l'injustice. Paris, Seuil.

Deranty J.-P., 2005. Les horizons marxistes de l'éthique de la reconnaissance. Actuel Marx, $\mathrm{n}^{\circ} 38$, p. 159-179.

Foucault M., 1975. Surveiller et Punir, Paris, Gallimard.

Foucault M., 1979. Naissance de la biopolitique. Annuaire du Collège de France, Histoire des systèmes de pensée, 1978-1979, 79e année, p. 367-372.

Haraway D., 2007. Manifeste cyborg et autres essais. Sciences-Fictions-Féminismes. Anthologie établie par Laurence Allard, Delphine Gardey et Nathalie Magnan, Paris, Exils-Essais.

Harvey D., 2008. La Géographie de la domination. Paris, Les Prairies ordinaires.

Herod A., 1997. From a geography of labor to a labor geography: labor's spatial fix and the geography of capitalism. Antipode, janvier, vol. 29, n 1, p. 1-31.

Honneth A., 2000. La lutte pour la reconnaissance, Paris, Cerf. 
Honneth A., 2006. La société du mépris. Vers une nouvelle Théorie critique. Edition établie par 0. Voirol, Paris, La Découverte.

Mitchell D., 2012. They saved the crops. Labor, landscape and the struggle over industrial farming in Bracero-era California (geographies of justice and social transformation $\mathrm{n}^{\circ} 10$ ). Athens, University of Georgia Press.

Mitchell D., 2011. Labor's geography: Capital, violence, guest workers and the post-world, war II landscape. Antipode, vol. 43, $\mathrm{n}^{\circ} 2$.

Renault E., 2004. L'Expérience de l'injustice. Reconnaissance et clinique de l'injustice. Paris, La Découverte.

Renault E., Zeneidi D., 2008. Formes de reconnaissance conflictuelle : relations sociales, appropriation de territoire, culture et politique dans un groupe de punks squatters. In Payet J.-P., Battegay A. (dir.), La reconnaissance à l'épreuve. Explorations socio-anthropologiques, Genève, Septenrion, p. 193-199.

\section{NOTES}

1. La recherche de Djemila Zeneidi a été soutenue financièrement par le programme ANR Terrferme (Les dispositifs de l'enfermement, approche territoriale du contrôle politique et social contemporain) coordonné par Bénédicte Michalon, chargée de recherche au CNRS - ADESS UMR 5185.

\section{AUTEUR}

\section{ALBENA TCHOLAKOVA}

Albena Tcholakova, albena.tcholakova@laposte.net, est sociologue au CNRS-CRESPPA, équipe GTM UMR 7217, post-doctorante GESTES. 